\title{
Extending Paxos/LastVoting with an Adequate Communication Layer for Wireless Ad hoc Networks *
}

\author{
Fatemeh Borran \\ Ecole Polytechnique Fédérale \\ de Lausanne (EPFL) \\ 1015 Lausanne, Switzerland \\ fatemeh.borran@epfl.ch
}

\author{
Ravi Prakash \\ University of Texas at Dallas \\ Department of Computer Science \\ Texas 75080-3021, U.S.A. \\ ravip@utdallas.edu
}

\author{
André Schiper \\ Ecole Polytechnique Fédérale \\ de Lausanne (EPFL) \\ 1015 Lausanne, Switzerland \\ andre.schiper@epfl.ch
}

\begin{abstract}
Most papers addressing consensus in wireless ad hoc networks adopt system models similar to those developed for wired networks. These models are focused towards node failures while ignoring link failures, and thus are poorly suited for wireless ad hoc networks. The recently proposed HO model does not have this drawback. The paper shows that an existing algorithm and the $\mathrm{HO}$ model can be used for multi-hop wireless ad hoc networks, if extended with an adequate communication layer. The description of the communication layer is augmented with simulation results that validate the feasibility of our approach and provide better understanding of the behavior of wireless environments.
\end{abstract}

\section{Introduction}

Ad hoc networks are self-organizing wireless networks that do not rely on a preexisting infrastructure to communicate. Nodes of such networks have limited transmission range, and packets may need to traverse multiple nodes before reaching their destination. Both process and link failures are possible. Packet loss is more frequent than traditional networks due to the collisions and channel interference. In wireless networks, an algorithm with high message complexity may lead to a high number of collisions, i.e., a high loss degree. In other words, it is even more important to have algorithms with low message complexity in wireless networks.

Consensus has been extensively studied in traditional networks with various system models. It is now well known that solving consensus deterministically requires some synchrony assumptions [13]. One option is to assume that the

\footnotetext{
${ }^{*}$ Research funded by the Swiss National Science Foundation under grant number 200021-111701, and the U.S. National Science Foundation under grant number 0437227.
}

(asynchronous) system eventually becomes synchronous, called partial synchrony [12]; another option is to augment the (asynchronous) system with failure detectors [8].

Starting from this background, some papers have considered the consensus problem in ad hoc networks. These papers essentially adopt system models similar to those developed for wired and static networks (sometimes with extensions), and these adoptations are not adequate for modelling ad hoc networks properly. Indeed, the models for wired networks are strongly biased towards node failures to the detriment of link failures. This bias has its root in the FLP paper [13], which assumes process crashes and reliable links. The bias was later strengthened by the failure detector model [8], which also assumes process crashes and reliable links. The bias is so commonly accepted that it is easily overlooked. However, overlooking the bias results in attempts to use solutions for environments where the bias is acceptable, to environments where the bias is unacceptable. This is the case with ad hoc networks, where assuming that links are reliable is clearly inappropriate. One may argue that if reliable links are required to solve a problem then there is no work-around, and reliable links need to be implemented on top of lossy links, even if this is expensive in ad hoc networks. But this is not the case for consensus. We know that consensus can be solved in a model in which the distinction between faulty processes and faulty links completely disappears, namely the Heard-Of (HO) model $[10,16,9]$. This model has no bias, and is, therefore, well suited to handle transient process and link faults. Not only transient link faults (message losses) are frequent in ad hoc networks, but transient process faults can also occur: consider a wireless device that temporarily becomes unavailable due to an obstacle to signal propagation.

Having said this, the goal of the paper is to show that an existing consensus algorithm can be used for ad hoc networks, if extended with an adequate communication layer. As suggested above, we believe that the right model for 
consensus in ad hoc networks is a model that handles process and link faults with the same mechanism, e.g., the HO model. Several consensus algorithms have been expressed in this model, see [10]. Out of these algorithms, only two of them genuinely tolerate message loss: the OneThird-Rule (OTR) algorithm, and the Paxos/LastVoting algorithm (LastVoting is basically Paxos [17] expressed in the HO model). OTR is certainly not adequate, because it is too costly in ad hoc networks ( $n$ - $n$ communication pattern, i.e., in every step all processes send messages to all) Paxos/LastVoting is based on the much more economical 1$n$ communication pattern (communication only between the coordinator and the other processes).

As in most papers about consensus in ad hoc networks, we assume that the number of nodes in the network, or at least an upper bound, is known (we comment on approaches that do not rely on this assumption in Section 2). Note that such an assumption is not unreasonable. Indeed, in most real wireless ad hoc networks nodes have to go through an admission before becoming operational. Also, several deployments of wireless sensors are planned deployments where a central entity makes decisions about how many and where to deploy the nodes.

Contribution: The paper shows that an adequate communication layer can nicely handle the 1- $n$ communication pattern in multi-hop networks without any additional overhead for the routing of messages or for election of the coordinator process. The description of the communication layer is completed with simulation results that validate the feasibility of our approach and provide better understanding of the behavior of realistic wireless environments.

Roadmap: The paper is organized as follows. Section 2 presents an overview of the related work. Section 3 presents the consensus algorithm and HO model. Section 4 describes the communication layer. Simulation results are presented in Section 5. Section 6 concludes the paper.

\section{Related work}

Several papers have addressed the consensus problem in wireless networks. One of the earliest solution to the consensus problem for a cellular network was proposed by Badache et al. [2]. The solution relies on a traditional fixed infrastructure of Mobile Support Station (MSS), and consensus is basically solved among the MSS using the Chandra-Toueg consensus protocol with the failure detector $\diamond \mathcal{S}$ [8]. The MSS then propagate the decision to the mobile hosts. The solution does not address mobility.

Vollset et al. [19] propose a family of broadcast protocols to be used for solving consensus using randomization. The communication pattern is $n$ - $n$. However, as pointed out in Section 1, the $n$ - $n$ communication pattern is not a good choice for multi-hop ad hoc networks. We believe that our
1- $n$ broadcast-convergecast algorithm is much more efficient than the general broadcast protocols proposed in [19].

Wu et al. [20], propose a consensus protocol for mobile ad hoc networks based on the failure detector $\diamond \mathcal{P}$. Wu et al. recognize the problem related to the reliable link assumption, but state that complicated design changes would be needed to enable their solution to work with lossy channels. In addition to the issue of using failure detectors in ad hoc networks, the solution has another weakness. It imposes a two-layer hierarchy on the network, where $k$ "predefined" nodes act as clusterheads. Each mobile node is associated with a clusterhead $(k<n)$. The solution tolerates up to $f$ faulty nodes, where $f<\min (k, n / 2)(f<k$ because the solution requires one correct clusterhead). If clusterheads change during the execution, then agreeing on the clusterheads involves solving consensus which leads to circularity.

Not knowing $n$ (called CUP, Consensus with Unknown Participants) has been considered by Cavin et al. [6]. The paper assumes that the identity and the number of the nodes participating in the consensus are unknown, but assumes reliable channels and nodes that never crash. The notion of participant detectors is introduced, and the paper establishes a necessary and sufficient condition on the participant detectors for solving consensus. Later [7] relaxes the requirement of non-faulty nodes, while Greve et al. [14] have extended the participant detectors of [6] to include node crashes. Channels need to be reliable.

Chockler et al. [11] developed a grid-based consensus algorithm with locally unknown participants in wireless ad hoc networks. The network is divided into a series of nonoverlapping grid squares, where each grid square is assumed to be populated. Every node knows a priori its location in the grid. Single-hop consensus is first run for each grid square and, then, all nodes gossip the local decisions. Once a node has received a value from every grid square, it can decide by applying a deterministic function to the set of values received (which requires that every grid square provides a value). Contrary to this solution, we do not require any clustering algorithm, we do not require nodes to know their position, and we do not modify the medium access control (MAC) layer implementation. Moreover the paper makes strong synchrony assumptions (inter-node communication delay are bounded by known constants), nodes are assumed not to crash in the middle of executing a broadcast instruction, and the model does not assume node recovery after a crash. In other words a rather complex system model is considered, in contrast to our very simple model.

\section{Consensus algorithm and HO model}

We consider a set $\Pi$ of processes. The consensus problem over a set $\Pi=\left\{p_{1}, p_{2}, \ldots, p_{n}\right\}$ of processes is defined by the following properties: 
- Validity: Any decision is the initial value of some process.

- Uniform Agreement: No two processes decide differently.

- Termination: All processes eventually decide. ${ }^{1}$

\subsection{The HO model}

For solving consensus, we consider the $\mathrm{HO}$ model defined in [10]. The model is based on (asynchronous) rounds. In a round every process first sends messages, then receives messages, and finally changes its state based on the set of messages received. We use the notation $H O(p, r)$ to denote the set of processes from which a message of round $r$ is received by process $p$ (heard-of set). Rounds are communication-closed, meaning that a message sent in round $r$ can only be received in round $r$. An algorithm expressed in the $\mathrm{HO}$ model is completed by a predicate over the collection of heard-of sets $(H O(p, r))_{p \in \Pi, r>0}$. For example, predicate $\forall r>0, \forall p \in \Pi:|H O(p, r)|>\lfloor n / 2\rfloor$ asserts that every heard-of set is a majority set. Consensus is solved in the HO model by a round-based algorithm together with an $\mathrm{HO}$ predicate, as shown in Section 3.2.

If for some round $r$, we have $q \notin H O(p, r)$, this means $p$ did not receive $q$ 's message of round $r$. This can be due to the crash of the sender $q$, or to a failure of the link between $p$ and $q$. The model does not need to distinguish between the two cases. This makes the model well suited to handle transient (process and link) faults [16] which are more general than crash-stop faults.

\subsection{The Paxos/LastVoting algorithm}

The Paxos/LastVoting algorithm [10] is the most appropriate algorithm for ad hoc networks (LastVoting is basically Paxos [17] expressed in the HO model, and is also close to the Chandra-Toueg $\diamond \mathcal{S}$ [8] consensus algorithm): its message complexity is $O(n)$, and it tolerates rounds $r$ in which $H O(p, r)$ is empty for all $p$, (i.e., it tolerates message loss). The code is given in Algorithm 1. From here on we call the algorithm simply LastVoting.

LastVoting consists a sequence of phases $\phi$, where each phase has 4 rounds ( $4 \phi-3$ to $4 \phi$ ). Each round $r$ consists of a sending step denoted by $S_{p}^{r}$ (sending step of $p$ for round $r$ ), and of a state transition step denoted by $T_{p}^{r}$. $\operatorname{Coord}(p, \phi)$, which denotes the coordinator of $p$ in phase $\phi$, is provided by the communication layer, see Section 4 . The communication layer also provides the messages received from the set $H O(p, r)$.

Before presenting our communication layer, we describe briefly how the LastVoting algorithm works: Each process $p$ has a timestamp $t s_{p}$ attached to its proposal $x_{p}$. (1) In

\footnotetext{
${ }^{1}$ Usually termination requires only "correct" processes to eventually decide. However, since we assume a model with transient faults, we consider a different termination property.
}

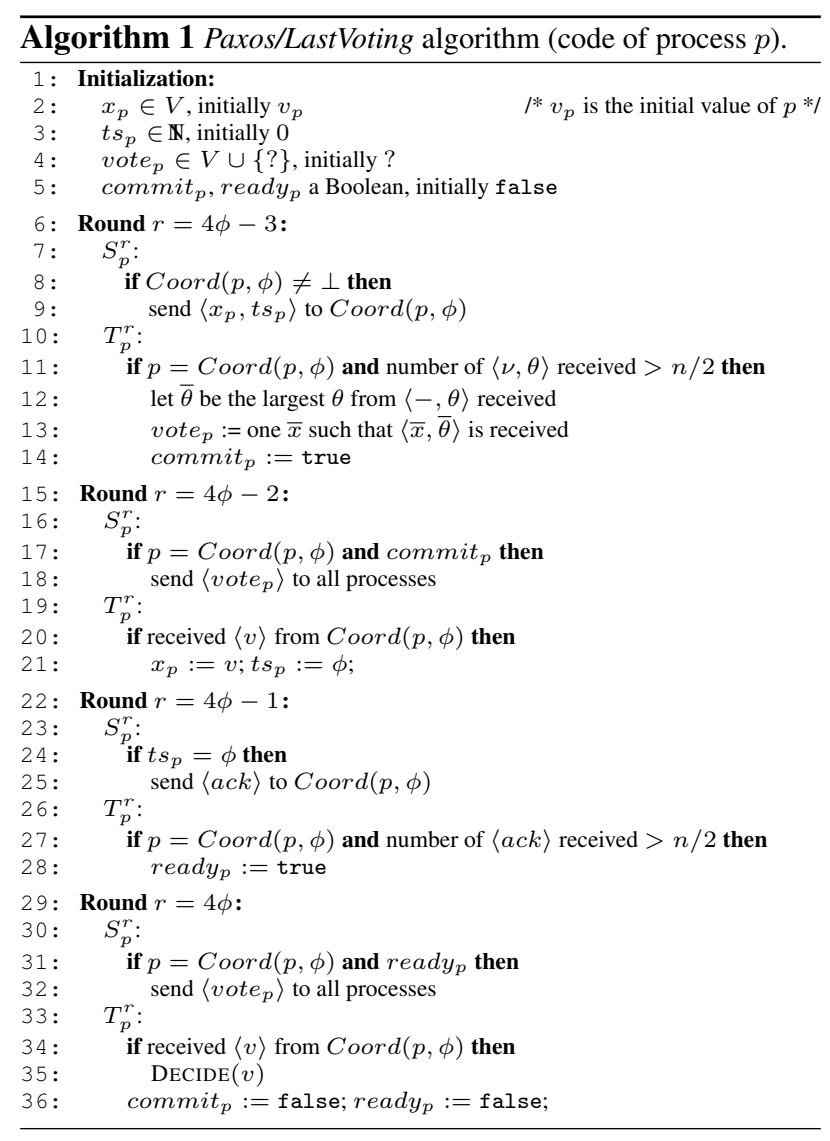

the first round of every phase, each process sends its proposal and timestamp to its coordinator (line 9). If the coordinator receives proposals from a majority of processes, it sets its vote to the last proposal with the highest timestamp (line 13). (2) In the second round, the coordinator sends its vote to all (line 18). Every process that receives coordinator's vote (line 20), changes its proposal and updates its timestamp. (3) These processes send an ack message to the coordinator in the third round (line 25). If the coordinator receives a majority of acks (line 27), it can decide on its vote: (4) the coordinator sends its vote (the decision) to all processes in the last round (line 32), and each process that receives the coordinator's vote decides (line 35 ).

The proof of Algorithm 1 can be found in [10]. The algorithm is always safe even if there are several coordinators per phase. The liveness of algorithm is ensured by the existence of a phase $\phi_{0}$ in which following predicate holds:

- A majority of processes consider the same coordinator $c_{0}$ in $\phi_{0}: \exists M \subseteq \Pi,|M|>n / 2, \forall p \in M: \operatorname{Coord}\left(p, \phi_{0}\right)=c_{0}$, - A majority of processes $p$ receive $c_{0}$ 's message in rounds $4 \phi_{0}-2$ and $4 \phi_{0}: \exists M \subseteq \Pi,|M|>n / 2, \forall p \in M$ : $c_{0} \in H O\left(p, 4 \phi_{0}-2\right)$ and $\exists M \subseteq \Pi,|M|>n / 2, \forall p \in M$ : $c_{0} \in H O\left(p, 4 \phi_{0}\right)$, and

- The coordinator receives the message from a majority of 
processes in rounds $4 \phi_{0}-3$ and $4 \phi_{0}-1$ : $\mid H O\left(c_{0}, 4 \phi_{0}-\right.$ $3) \mid>n / 2$ and $\left|H O\left(c_{0}, 4 \phi_{0}-1\right)\right|>n / 2$.

\section{Communication layer for LastVoting}

The communication layer's role is to ensure the above predicates, which includes the election of a coordinator.

\subsection{System model}

Wireless network: We consider an asynchronous multihop wireless network consisting of set of $n$ nodes. ${ }^{2}$ We use the terms node and process interchangeably. Each node in the network has a single wireless transceiver through which it can communicate with other nodes. The maximum distance at which a node's transmission can be successfully received may be less than the upper bound on the communication range. Moreover, this distance may change from one transmission to the next. This is different from the unit-disk graph model, and a more realistic representation of wireless propagation characteristics.

Unreliable links and unpredictable delays: When employing MAC layer broadcast, the transmitter does not necessarily know the identities of all nodes within its communication range. Nor does the transmitter know the subset of nodes that successfully received the message. Broadcast communication satisfies the basic integrity and no-duplication properties guaranteeing that every received message was previously broadcast, and each message is received at most once. However, it is inherently unreliable: the receivers do not send any acknowledgment, and the sender does not make any retry attempts to increase the likelihood of message delivery to neighbors. Though MAC layer unicast is described as being reliable (uses acknowledgments), there is no guarantee that a data frame will be forwarded to the intended neighbor. So, we assume that the wireless links are unreliable and the message communication delay is unpredictable: our algorithm doesn't require any protocol like TCP, unlike [20].

Node crashes: In addition to link failures, nodes can crash. Faults can be transient or permanent, but a majority of nodes must remain connected despite permanent faults. Note that this does not prevent nodes from being temporarily disconnected.

Good period: LastVoting is always safe. To ensure liveness, we must restrict the asynchrony of the system. We assume that, from time to time, unknown to the processes, the system experiences good periods, during which messages are reliably transmitted with the end-to-end (multihop) transmission delay bounded by a known constant $\delta .{ }^{3}$

\footnotetext{
${ }^{2}$ Actually $n$ needs only to be an upper bound of the number of nodes.

${ }^{3}$ It would be easy to adapt the algorithm to an unknown $\delta$ value [12], e.g., using adaptive timeout.
}

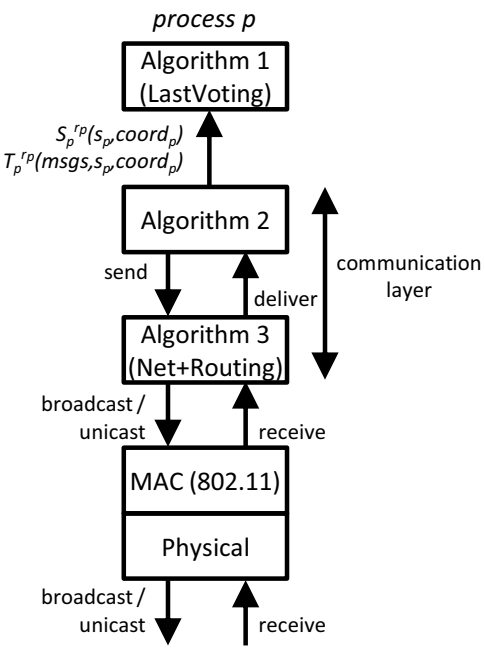

Figure 1. Architecture.

Note that this is not in contradiction with our previous assumption about unreliable links and unpredictable delays. This is required to overcome FLP impossibility result. The notion of good period is a more realistic system assumption than partially synchronous systems, inspired from [12] and already used in [16].

\subsection{Architecture}

Figure 1 shows the overall view of our architecture. The uppermost layer corresponds to LastVoting (Algorithm 1). Algorithm 1 contains two functions $S_{p}^{r}$ and $T_{p}^{r}$ that are called by the layer beneath, namely Algorithm 2:4

- The sending step $S_{p}^{r}$ of Algorithm 1 is a function $S_{p}^{r}\left(s_{p}, \operatorname{coor}_{p}\right)$ that takes as input the round number $r$, the state $s_{p}$, the coordinator $\operatorname{coord}_{p}$, and returns the set of message(s) $m s g$ to be sent, together with their destination(s) dst (see Algorithm 2, line 15).

- The state transition step $T_{p}^{r}$ of Algorithm 1 is a function $T_{p}^{r}\left(m s g s, s_{p}, \operatorname{coord}_{p}\right)$ that takes as input the round number $r$, the set of messages received ( $m s g s$ ), the state $s_{p}$, the coordinator $\operatorname{coord}_{p}$, and returns the new state $n s_{p}$ (see Algorithm 2, line 32).

Algorithm 2 uses Algorithm 3 as a simple and best-effort broadcast and convergecast algorithm on top of the MAC sub-layer, which typically uses a CSMA/CA-based protocol like IEEE 802.11. For sending a message, Algorithm 2 calls the send function of Algorithm 3. Upon reception of a message by Algorithm 3, the deliver function of Algorithm 2 is called. Both MAC layer broadcasts and unicasts are used by Algorithm 3: when a message has to be locally broadcast, the MAC layer broadcast primitive is used.

\footnotetext{
${ }^{4}$ Actually Algorithm 1 does not send the messages in lines 9, 18, 25, 32 ; it simply defines which messages should be sent to which destinations.
} 


\subsection{Algorithm 2: the upper communication layer}

For every process $p$, Algorithm 2 has two main roles:

- Elect the coordinator (to be used as a parameter of the $S_{p}^{r}$ function).

- For every round $r$, construct the set of messages received by $p$ (to be used as a parameter of the $T_{p}^{r}$ function).

Before discussing these two issues, some general explanations are needed. First, note that Algorithm 2 handles the process state $s_{p}$ (line 3), the phase number $\phi_{p}$ (line 8 ) and the round number $r_{p}$ (line 14). Second, Algorithm 2 relies on Algorithm 3 for sending (and receiving) messages (e.g., line 17): the routing implemented by Algorithm 3 is optimized to drop unnecessary messages. Third, Algorithm 2 is designed to ensure fast phase synchronization once a good period has started. Phase synchronization is needed, since when a good period starts, processes can be in different phases (and different rounds). Fast phase synchronization means that processes quickly join the same phase, in order to allow processes to decide. This is done as follows: Each process attaches its current phase number $\phi_{p}$ and round number $r_{p}$ to the messages it sends (e.g., line 17). Whenever a process receives a message from some phase $\phi>\phi_{p}$, jumps to the first round of that phase (line 31,12 ).

Coordinator election: Each process has a priority (e.g., the process identity, line 5), and the process that believes to have the highest priority for some phase $\phi$ becomes the coordinator for that phase. To be more efficient, the coordinator is restricted to a predefined set Contender $\subset \Pi^{5}$ Initially, every process $p \in$ Contender considers itself as a coordinator (line 4).

At the beginning of each phase $\phi$, every process $p$ that considers itself to be coordinator, sends its identity and priority to all (line 11). This is the only message that Algorithm 2 sends in addition to the messages of Algorithm 1. Each process $p \in \Pi$ that receives a message from phase $\phi \geq \phi_{p}$ from some process $q$ with higher priority (line 23, 28 ), updates its coordinator to $q$ and priority to $q$ 's priority.

After the beginning of a good period, let $\tau$ be the time at which the first process starts some phase $\phi_{0}$ (other processes are in earlier phases: with smaller phase numbers). Then at time $\tau+2 \delta$ there is a unique coordinator $c$ for all phases $\geq \phi_{0} \cdot{ }^{6}$ However, a unique coordinator $c$ at time $\tau+2 \delta$ is not enough to ensure termination in phase $\phi_{0}$ : multiple coordinators between $\tau$ and $\tau+2 \delta$ can prevent a decision in phase $\phi_{0}$. So phase $\phi_{0}+1$ is started after $2 \delta$ in case $c$ is still in round $4 \phi_{0}-3$ (line 37 ); $c$ is the unique coordinator for the remainder of the good period.

\footnotetext{
${ }^{5}$ The Contender set must be large enough to ensure that all its members are not crashed at the same time.

${ }^{6}$ All proofs are in the Appendix of [4].
}

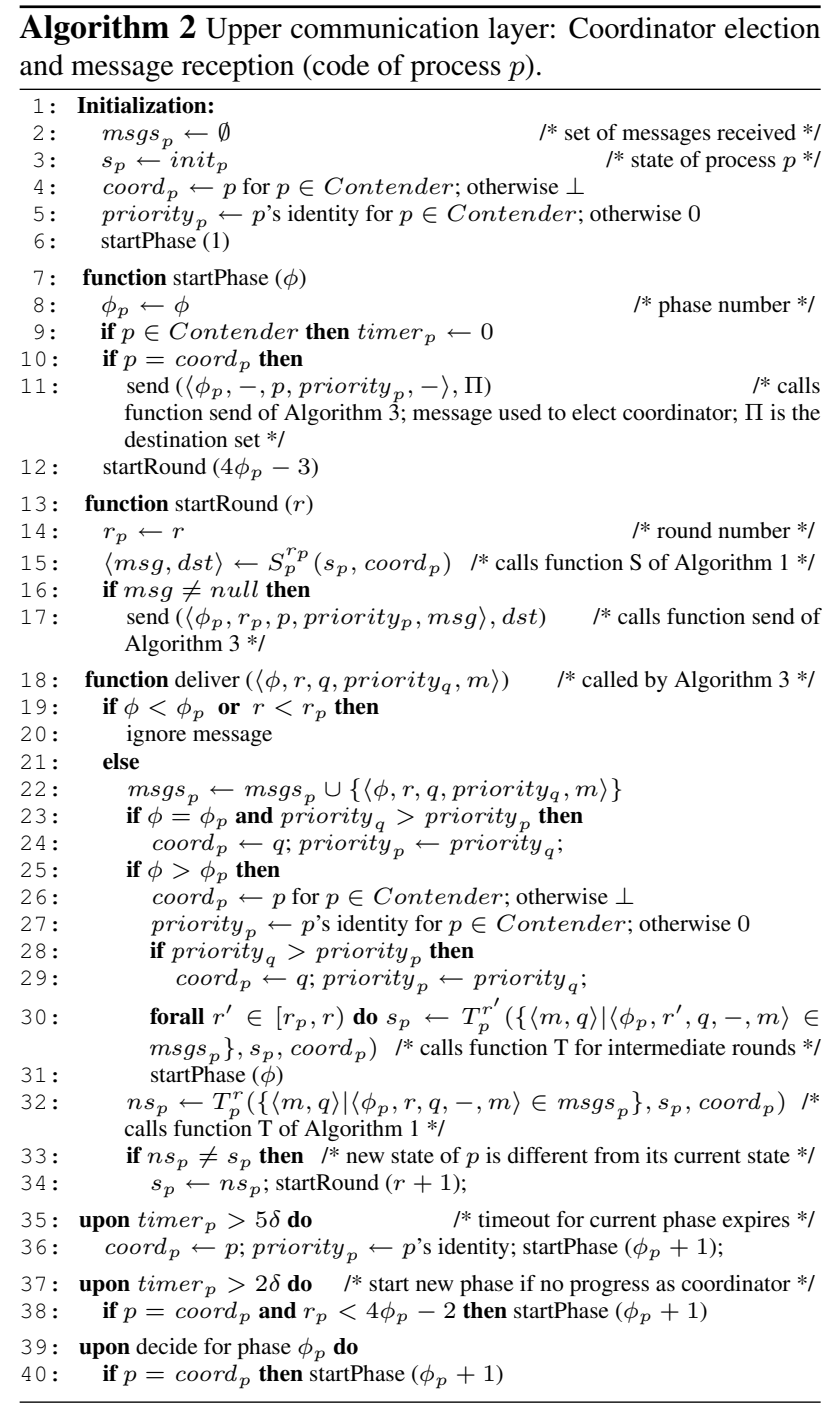

Message reception: For every round $r$, Algorithm 2 constructs the set of messages received by process $p$ (to be used as a parameter of the $T_{p}^{r}$ function). This is done differently whether $p \in$ Contender or $p \notin$ Contender. If $p \notin$ Contender, then $p$ does not use a timer; if $p \in$ Contender then $p$ uses a timer.

Case 1: $p \notin$ Contender. In this case $p$ remains in the current round $r_{p}$ of phase $\phi_{p}$ until (1) it receives a message from a larger phase (line 25) or (2) $p$ has received "enough" messages in round $r$ (lines 32 to 34). Note that Algorithm 2 does not know what "enough" means. "Enough" is defined by Algorithm 1: in rounds $4 \phi-3$ and $4 \phi-1$ "enough" is more than $n / 2$; in rounds $4 \phi-2$ and $4 \phi$ "enough" is 1 . The solution is for Algorithm 2 to call the $T_{p}^{r}$ function whenever a new message is received (line 32 ): if not enough messages have been received, the $T_{p}^{r}$ function does not modify the state (line 33) and $p$ remains in the same round (in order to 
wait for more messages).

Case 2: $p \in$ Contender. In addition to behaving like an ordinary process (Case 1), $p$ uses a timer, which is reset at the beginning of each phase $\phi_{p}$ (line 9). In a good period a round does not take more than $\delta$. So, in addition to the behavior explained under Case $1, p$ remains in phase $\phi_{p}$ until (1) $2 \delta$ time units have elapsed (duration of coordinator election round and round $4 \phi-3$ ) and $p$ is still in round $4 \phi_{p}-3$ (line 37), or (2) $5 \delta$ time units have elapsed (duration of coordinator election round and rounds $4 \phi-3$ to $4 \phi$ ) and $p$ is still in phase $\phi_{p}$ (line 35).

Optimizations: Algorithm 2 includes two optimizations. The first one is useful when several instances of consensus are running one after the other (e.g., atomic broadcast). When a decision occurs in phase $\phi$, the coordinator starts immediately phase $\phi+1$ (line 39) without waiting the timeout for phase $\phi$. The second optimization avoids unnecessary coordinator changes. Once some process $p$ is considered to be the coordinator by a majority, it remains the coordinator as long as its messages reach a majority of processes: process $q \in$ Contender that considers $p$ as its coordinator (priority $_{q}<$ priority $_{p}$ ) does not change its coordinator unless its timer expires (line 35).

\subsection{The lower communication layer: broad- cast and convergecast}

Algorithm 2 invokes Algorithm 3 (lower communication layer) when it sends a message in lines 11 and 17. Depending on dst, Algorithm 3 uses diffusion or convergecast in lines 8 and 10: diffusion is used for a message sent by a coordinator (1 to all), while convergecast is used for messages sent to the coordinator (all to 1). Diffusion messages are identified by the tag MESSAGE (e.g., line 8), while convergecast messages are identified by the tag RESPONSE (e.g., line 10). During diffusion, Algorithm 3 delivers the message that is received for the first time (line 12) to Algorithm 2. During convergecast, the message is delivered only if it reaches its destination (line 18). Algorithm 3 also contributes to an efficient election of the coordinator by discarding messages from contenders that can no more become coordinator.

Diffusion: As all participating nodes are not within communication range of each other, it is not possible for a node to directly communicate with others. Hence, a networkwide message broadcast can be implemented through diffusion. The message source (a coordinator) will broadcast the message locally at the MAC layer (line 8). When node $p$ receives a message from some node $q$ for the first time (line 11), it becomes a child of $q$ (line 14) only if priority $_{q}>$ priority $_{p}$ ( $q$ wins against $p$ in the election). Then it broadcasts the message at the MAC layer (line 16) except when priority $_{q}<$ priority $_{p}$ ( $q$ loses against $p$ in the
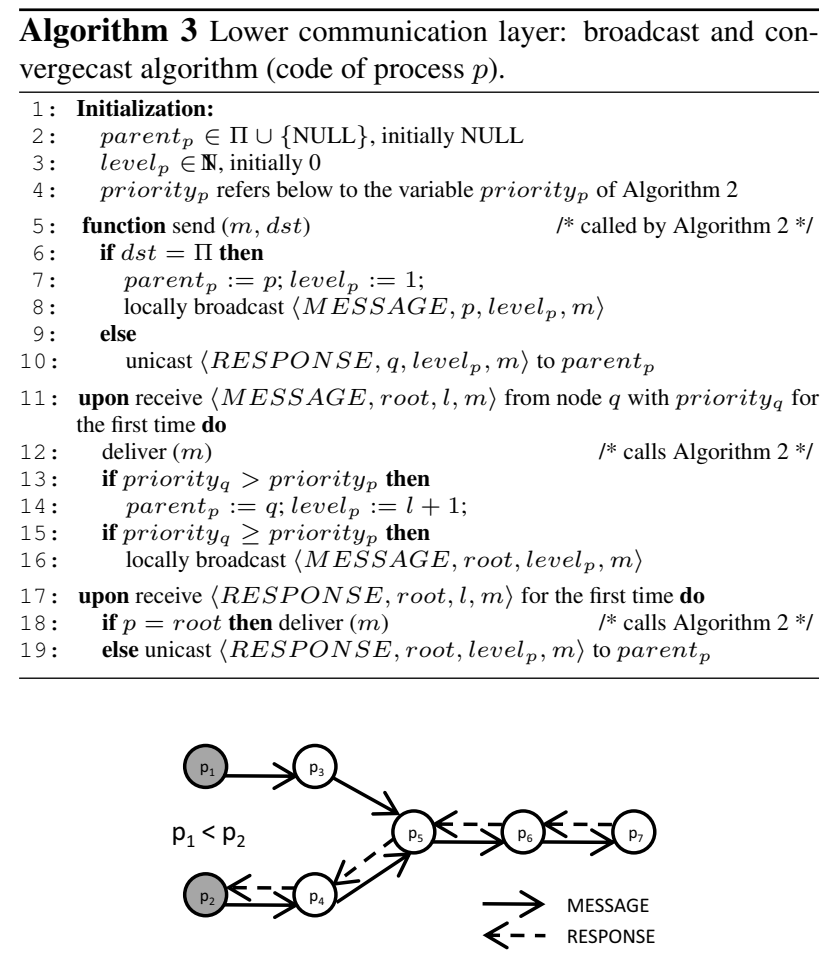

Figure 2. Broadcast $v s$. convergecast.

election). When a node receives copies of the same message later, it ignores them. As a result, an efficient tree rooted at a coordinator is formed.

Convergecast: The tree constructed during diffusion is used by convergecast, to transport responses to the coordinator, the root of the tree. As a node does not know the identities of all its children, it is not possible for the node to determine when it has received responses from all of them. Therefore, each node sends its response to its parent as soon as the node joins the tree. Subsequently, whenever the node receives a response from any child it forwards the received response to its parent. Figure 2 shows an example of broadcast and convergecast protocol in a multi-hop network. During diffusion (tag MESSAGE), since $p_{2}$ 's priority is higher than $p_{1}$ 's, if $p_{5}$ receives the message form $p_{2}$ before $p_{1}$, it ignores $p_{1}$ 's message. Otherwise, it diffuses both, but $p_{4}$ becomes its parent and $p_{2}$ its grand parent. During convergecast (tag RESPONSE), only path from $p_{7}$ to $p_{2}$ is followed.

Gradient-based convergecast: If any node on the path from node $p$ to the root of the tree (i.e., to the coordinator) is down, or any link on this path is lossy, $p$ 's message may not reach the root. Gradient-based convergecast can increase the probability of responses reaching the root. During diffusion, as a node joins the tree, it sets its level to be one greater than its parent's level (line 14). The root is always at level one (line 7). During convergecast nodes listen to 
transmissions in the promiscuous mode. If they receive a message from a neighboring node at a higher level they retransmit the message (using MAC layer broadcast). Thus, messages travel from higher level to lower level, with no cyclic forwarding, ultimately reaching the root. Even if the path from the root to a node breaks down after the node has joined the tree, it may be possible for the node's response to reach the root along other gradient-based paths, if such paths exist. This can be done as follows:

1. In line 10, instead of sending the RESPONSE to the parent, locally broadcast the RESPONSE.

2 . In line 19 , first determine if $l>$ level $_{p}$. If so, locally broadcast the RESPONSE.

\section{Simulation}

We used JiST/SWANS v1.0.6 [1, 3] wireless network simulator. We consider a $m \times m$ square grid with nodes placed at each intersection as illustrated in Figure 3. The grid-based placement is used instead of the random uniform placement only for manageability reasons. For instance, using this placement we can select exactly which nodes belong to the Contender set. Communication between two nodes $p_{1}$ and $p_{2}$ occurs in an ad hoc manner using unicast/broadcast as defined in the IEEE 802.11b standard [15]. The data rate of the wireless channel is $1 \mathrm{Mbps}$. All nodes have the same transmission range $(150 \mathrm{~m})$. We modify the network area to vary network density and network diameter. Nodes are stationary, except for one case in which we measure the impact of mobility (see Section 5.2.5). We measure the impact of location and number of contenders in Section 5.2.3. Each contender starts the algorithm randomly between 0 and 10 milliseconds after simulation start time. The simulation lasts for 100 seconds. Every consensus packet is around 32 bytes.

Note that the IEEE 802.11b MAC layer specification uses CSMA/CA and enforces RTS/CTS/ACK control frames for unicast communication only. Collision control for broadcast is limited to basic collision avoidance carrier sensing, and broadcast is therefore prone to packet collisions. A straightforward approach to reduce collisions is to have nodes wait for a small random amount of time (jitter) before rebroadcasting.

Given the consensus algorithm in Section 3.2 and based on broadcast and convergecast protocol (Algorithm 3), we are interested in analyzing whether the required liveness condition is provided by Algorithm 2 and 3 in wireless ad hoc networks. The network that we consider is quite dense to avoid partitioning as much as possible and quite noisy (due to frequent collisions, node interference, and background traffic explained later) to simulate bad periods.

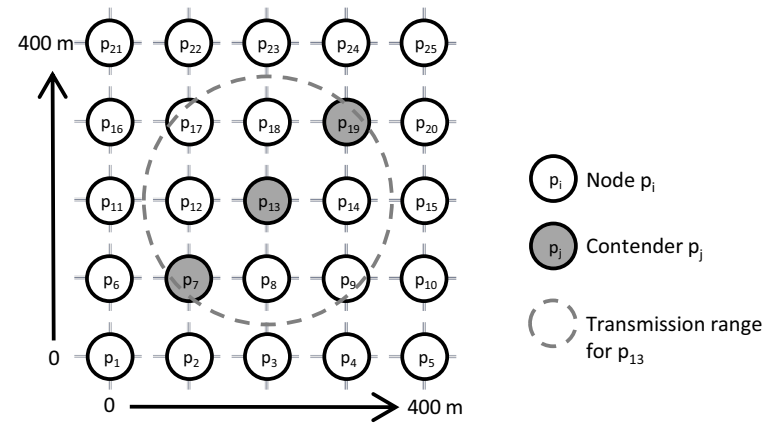

Figure 3. Square grid of size $5 \times 5$ in network area $400 \times 400 \mathrm{~m}^{2}$.

\subsection{Metrics}

In order to evaluate the performance of LastVoting consensus algorithm, several instances of consensus are run one after the other. Each process starts a new instance of consensus with new proposition. A new consensus is started as soon as the decision for current consensus is reached or a message from a later invocation of consensus algorithm is received. In the latter case, the previous decisions can be communicated through piggy-backing.

We have defined two (independent) metrics: consensus latency and consensus throughput. Consensus latency is expressed in terms of average number of phases per consensus from initialization to first decision. Consensus throughput represents how many instances of consensus can be run successfully in simulation time (100 seconds): the time for one consensus is simply 100/throughput seconds.

\subsection{Results}

We evaluate the performance of our consensus algorithm in both single and multi-hop networks. In these scenarios no process crashes and no packet is explicitly dropped: the only source of failure is the collisions and node interferences. ${ }^{7}$ However, to observe the performance of our algorithm in realistic situations, we added a background traffic to the system: every second, each node sends a packet (with the same size as consensus packet) to a random destination. We have noticed that increasing background traffic only reduces the throughput of our algorithm slightly (all additional graphs can be found in the Appendix of [4]). All results of simulations are averaged over 30 independent runs. The vertical bars in the graph represent $95 \%$ confidence interval for the mean.

First, we ran a calibration test to examine the behavior of the simulator and our routing algorithm to tune the amount

\footnotetext{
${ }^{7}$ Considering only message loss does not make consensus easier to solve: consensus is impossible to solve in a synchronous system with lossy links [18]. To solve consensus, message loss must be restricted.
} 


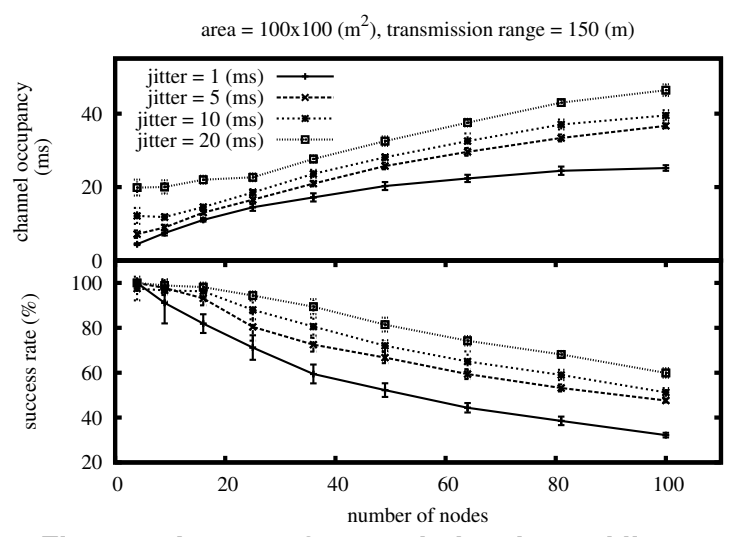

Figure 4. Impact of network density and jitter.

of the jitter. Figure 4 (top) shows once a single message has been broadcast, the duration for which the wireless channel remains busy (henceforth, referred to as channel occupancy duration). Note that the same message forwarding algorithm is employed by each node: on receiving a message for the first time, a node rebroadcasts the message after a random wait between 0 and jitter. So, the wireless channel becomes idle either when the message is received by everyone or is completely lost. For instance, for 100 nodes within range of each other, with jitter $=10 \mathrm{~ms}$, channel occupancy is $40 \mathrm{~ms}$. This gives us $200 \mathrm{~ms}$ for one phase of our consensus implementation $(5 \times 40 \mathrm{~ms})$. Figure 4 (bottom) shows the percentage of nodes that receive the broadcast message. It seems that the value of the jitter is optimal around 10 ms. With $10 \mathrm{~ms}$, at least a majority of processes have received the message and there is almost the same channel occupancy as $5 \mathrm{~ms}$. For the rest of simulations we fix jitter to $10 \mathrm{~ms}$.

5.2.1. Single-hop scenarios. First, we consider a single-hop network in which all nodes are in communication range of each other. The network area is $100 \times 100 \mathrm{~m}^{2}$. We gradually increased the network density. Only a single node, for example $p_{1}$, belongs to the Contender set. We measured the average number of phases per consensus in networks with different node densities (from 4 nodes to 100 nodes) by varying the timeout. The value of timeout refers to $5 \delta$ used in Algorithm 2. The ideal value in our scenario is 1 phase per consensus. However, this value can increase in the presence of packet loss.

Figure 5 (top) shows how the number of required phases varies with timeout. Logarithmic scales are used in $\mathrm{x}$-axis to better visualize a large range of timeout and emphasize the small timeouts. Beyond a certain value of timeout, the number of phases to terminate consensus remains almost constant (1 phase) as density of the deployment increases. Figure 5 (bottom) shows how consensus throughput varies with timeout for several network densities. Note that the results

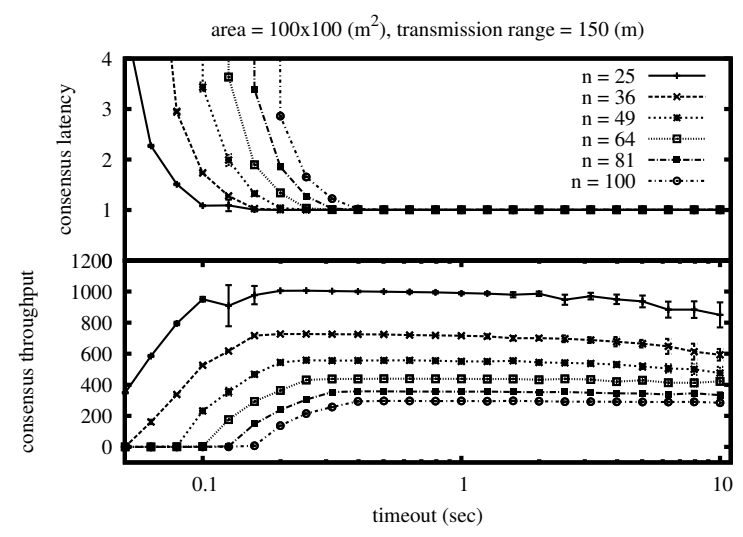

Figure 5. Impact of timeout in single-hop networks.

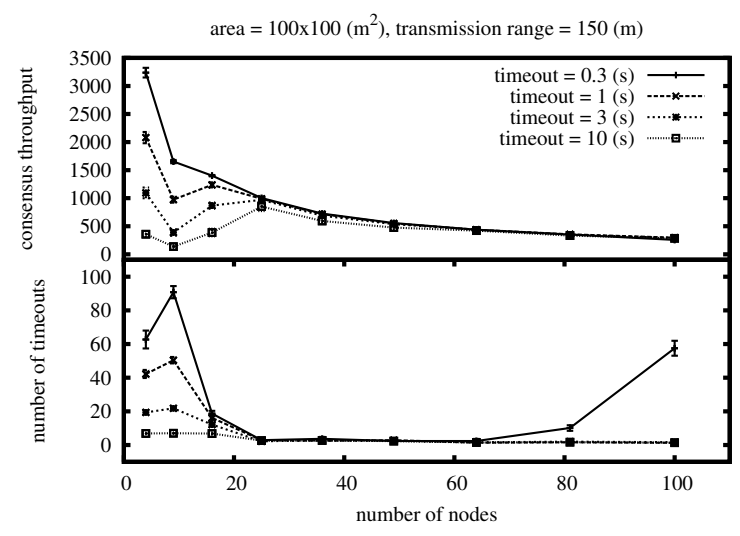

Figure 6. Impact of density in single-hop networks.

we have obtained in this simulation based on the timeouts confirm our previous results on channel occupancy (e.g., for $n=64$ the best throughput is for timeout $\approx 200(\mathrm{~ms})$ which is greater than $5 \times 30(\mathrm{~ms})$ taken from Figure 4 (top)).

Figure 6 (top) is just another representation of Figure 5 (bottom) to better visualize the impact of network density. In general, by increasing density (number of nodes), the throughput of our algorithm decreases, independent of phase timeout value. This is because of message losses due to increased collisions. The graph shows that there is an optimal value for density. After around 25 nodes, the throughput always goes down. So the algorithm performs less efficiently in the presence of more than 25 nodes per $10000 \mathrm{~m}^{2}$ (single-hop). Although with small number of nodes the throughput is high, the number of timeouts that occur is also high (see Figure 6 (bottom)). For instance, for $n=4$ the algorithm allows only one message loss while for $n=100,49$ losses are allowed in a round (majority set). This explains why for small number of nodes, increasing the timeout reduces the performance in Figure 6 (top). 


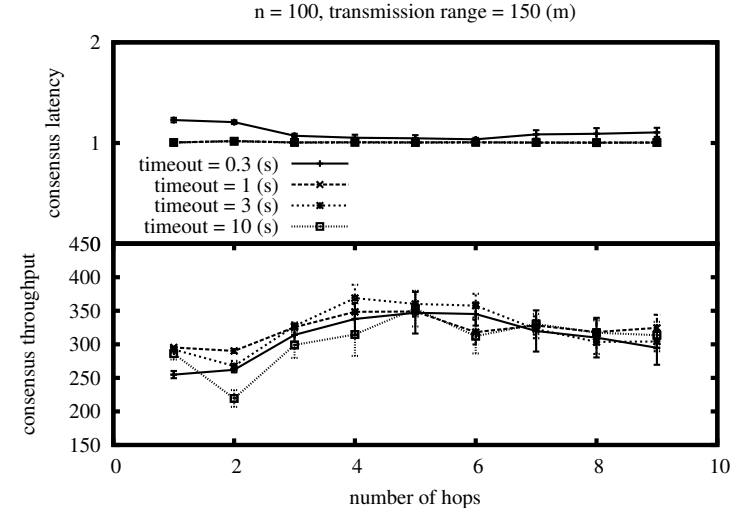

Figure 7. Impact of network diameter in multi-hop networks.

5.2.2. Multi-hop scenarios. We consider 100 nodes distributed in a $10 \times 10$ square grid. The transmission range for each node is fixed to $150 \mathrm{~m}$. To obtain multi-hop scenarios, we varied the network area from $100 \times 100 \mathrm{~m}^{2}$ (singlehop) to $900 \times 900 \mathrm{~m}^{2}$ (9-hops), and we chose $p_{1}$ as the coordinator ( $p 1$ is located at the lower left corner of the grid).

Figure 7 (top) shows the scalability of our algorithm in multi-hop networks. By increasing the network area for 100 nodes, on one hand we increase the number of hops and on the other hand we decrease the density and, therefore, the probability of message collisions. Figure 7 (bottom) shows the trade-off between number of hops and network density. From one-hop to four-hops, we decrease the density, so the performance is improved. From six-hops on, since the message must traverse more hops the performance is slightly decreased. So, 100 nodes perform better in five-hops. This gives approximately 20 nodes per hop. This is almost the same conclusion that we had from single-hop scenarios.

\subsubsection{Impact of location and number of con-} tenders. We varied the position of the contender (coordinator) from bottom-left corner to the center. ${ }^{8}$ We run a Kruskal-Wallis non-paired data test [5] (generalized Wilcoxon Rank Sum test) to determine if the position of the contender influences consensus throughput (null hypothesis: position of the contender does not influence consensus throughput). The test accepts the null hypothesis with p-value 0.9699. The conclusion is that the throughput of our consensus algorithm is independent of the contender's position. This seems reasonable in single-hop networks. In multi-hop networks, when the contender moves from bottom-left corner to the center of square grid, the number of hops from the contender to the farthest node is reduced while the number of collision is augmented (in center there

\footnotetext{
${ }^{8}$ This is enough to explore other possibilities because of the symmetry of square grid.
}

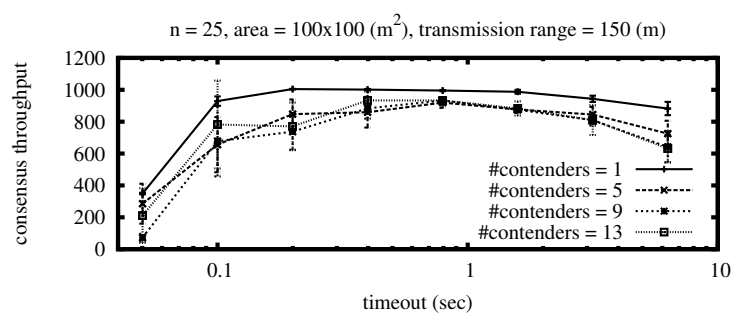

(a) Single-hop network

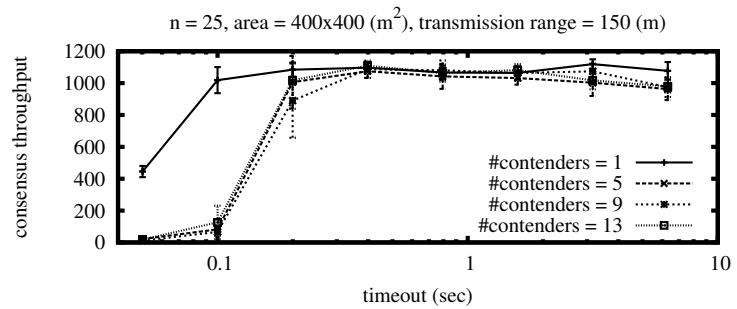

(b) Multi-hop network

Figure 8. Impact of contenders.

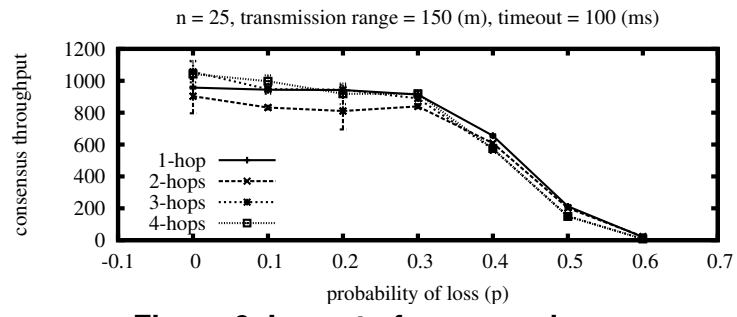

Figure 9. Impact of message loss.

is 4 times more collision than in corner). So in multi-hop networks, reduced number of hops is compensated by increased number of collisions.

In Figure 8, we increased the number of contenders in a network of 25 nodes from $1 \%$ to $50 \%$. The figure confirms that for large enough timeout, the number of contenders does not have an important impact on the consensus throughput. In fact, once the process with highest priority is elected as the coordinator, it remains the same as long as a majority of its messages are not lost.

5.2.4. Impact of message loss. We now consider scenarios in which a node on receiving a message can discard it with probability $p$ (uniform distribution). This simulates the loss of the message during its passage through the network. There is one coordinator located at the lower left corner of the grid. Figure 9 shows the sensitivity of our algorithm to $p$ and confirms the ability of our algorithm to tolerate a minority message loss.

5.2.5. Impact of mobility. Finally, we measure the impact of mobility on consensus throughput. We use the random waypoint model with a fixed speed and zero pause 


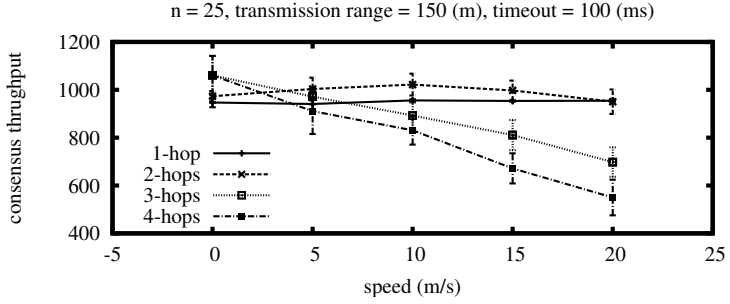

Figure 10. Impact of mobility.

time. In this model, nodes select an arbitrary destination in the field and move directly towards it at constant speed. When they reach the destination, they pick a new destination and so on. Figure 10 shows the behavior of our algorithm with node speed. The coordinator is located at the lower left corner of the grid at the beginning of the simulation, and then moves. Note that when the network diameter is 2 (network area is $200 \times 200$ ), a majority of nodes (13 nodes) are in communication range with the coordinator, which explains why there is no difference between 1-hop and 2-hop scenarios.

\section{Conclusion}

The Paxos/LastVoting algorithm extended with an adequate communication layer can potentially solve the consensus problem in wireless mobile networks. Paxos/LastVoting is safe by design, but a communication predicate is required to ensure the termination of consensus. We have proposed an appropriate implementation that satisfies the required communication predicate in good periods. We have validated our implementation by running simulations in multi-hop wireless networks. The results of simulations validate the existence of the good periods and confirm that our approach is applicable for realistic networks.

We could not compare our results with Chockler's paper [11] since they do not provide the time unit in their figures. The results in Vollset's paper [19] are far from being efficient (they require around 100 seconds in average for one instance of consensus). Finally, the performance evaluation in Wu's paper [20] is of limited utility since they do not use a realistic MAC layer in their simulations. Although the results of our paper are limited to the simulations, we believe that this approach is applicable in real systems. Our future work is to explore deployment of the system using a network of actual nodes.

Acknowledgments We would like to thank Nuno Santos, Paul Ezhilchelvan, and anonymous reviewers for their comments.

\section{References}

[1] JiST/SWANS. http://jist.ece.cornell.edu.
[2] N. Badache, M. Hurfin, and R. Macedo. Solving the Consensus Problem in a Mobile Environment. In IPCCC'99, Phoenix, Arizona, USA, February 1999.

[3] R. Barr. An efficient, unifying approach to simulation using virtual machines. $\mathrm{PhD}$ thesis, Cornell University, Ithaca, NY, May 2004.

[4] F. Borran, R. Prakash, and A. Schiper. Consensus in Wireless Ad hoc Networks. Technical Report LSR-REPORT-2008001, EPFL, 2008.

[5] J.-Y. L. Boudec. Methods, practice and theory for the performance evaluation of computer and communication systems, 2006. Lecture Notes, EPFL, Switzerland.

[6] D. Cavin, Y. Sasson, and A. Schiper. Consensus with Unknown Participants or Fundamental Self-Organization. In ADHOC-NOW 2004), pages 135-148, 2004.

[7] D. Cavin, Y. Sasson, and A. Schiper. Reaching Agreement with Unknown Participants in Mobile Self-Organized Networks in Spite of Process Crashes. Technical Report IC/2005/026, EPFL, 2005.

[8] T. D. Chandra and S. Toueg. Unreliable failure detectors for reliable distributed systems. J. ACM, 43(2):225-267, Mar. 1996.

[9] B. Charron-Bost and A. Schiper. Harmful dogmas in fault tolerant distributed computing. ACM SIGACT news, 38(1):53-61, March 2007.

[10] B. Charron-Bost and A. Schiper. The Heard-Of model: Computing in Distributed Systems with Benign Failures. Technical report, EPFL, June 2007.

[11] G. Chockler, M. Demirbas, S. Gilbert, C. Newport, and T. Nolte. Consensus and collision detectors in wireless ad hoc networks. In PODC '05, pages 197-206, New York, NY, USA, 2005. ACM.

[12] C. Dwork, N. Lynch, and L. Stockmeyer. Consensus in the presence of partial synchrony. J. ACM, 35(2):288-323, Apr. 1988.

[13] M. J. Fischer, N. A. Lynch, and M. S. Paterson. Impossibility of distributed consensus with one faulty process. J. ACM, 32(2):374-382, Apr. 1985.

[14] F. Greve and S. Tixeuil. Knowledge connectivity vs. synchrony requirements for fault-tolerant agreement in unknown networks. In DSN '07, pages 82-91, Washington, DC, USA, 2007. IEEE Computer Society.

[15] I. W. Group. Wireless LAN Medium Access Control (MAC) and Physical Layer (PHY) Specifications. IEEE Strandard 802.11-1997, New York, NY, 1997.

[16] M. Hutle and A. Schiper. Communication predicates: A high-level abstraction for coping with transient and dynamic faults. In DSNO7, pages 92-10. IEEE, June 2007.

[17] L. Lamport. The part-time parliament. ACMTCS, 16(2):133169, May 1998.

[18] N. Santoro and P. Widmayer. Time is not a healer. In Proceedings of the 6th Symposium on Theor. Aspects of Computer Science, pages 304-313, Paderborn, Germany, 1989.

[19] E. W. Vollset and P. D. Ezhilchelvan. Design and performance-study of crash-tolerant protocols for broadcasting and reaching consensus in manets. In SRDS '05, pages 166-178, Washington, DC, USA, 2005. IEEE Computer Society.

[20] W. Wu, J. Cao, J. Yang, and M. Raynal. Design and Performance Evaluation of Efficient Consensus Protocols for Mobile Ad Hoc Networks. IEEE Transactions on Computers, 56(8):1055-1070, August 2007. 